\title{
Performances épuratoires d'une filière de trois bassins en série de lagunage à microphytes sous climat sahélien : cas de la station de traitement des eaux usées de 21E (groupe EIER-ETSHER)
}

\author{
Performance of a series of three wastewater stabilisation \\ ponds in Sahelian climate: Case study of 21E (EIER-ETSHER) \\ wastewater treatment plant
}

\author{
Amadou Hama Maiga, Yacouba Konate, Joseph Wethe, Kokou Denyigba, Denis \\ Zoungrana et Lassana Togola
}

Volume 21, numéro 4, 2008

Numéro publié dans le cadre de l'Année internationale de l'assainissement

URI : https://id.erudit.org/iderudit/019163ar

DOI : https://doi.org/10.7202/019163ar

Aller au sommaire du numéro

Éditeur(s)

Université du Québec - INRS-Eau, Terre et Environnement (INRS-ETE)

ISSN

0992-7158 (imprimé)

1718-8598 (numérique)

Découvrir la revue

Citer cet article

Maiga, A. H., Konate, Y., Wethe, J., Denyigba, K., Zoungrana, D. \& Togola, L. (2008). Performances épuratoires d'une filière de trois bassins en série de lagunage à microphytes sous climat sahélien : cas de la station de traitement des eaux usées de 21E (groupe EIER-ETSHER). Revue des sciences de l'eau / Journal of Water Science, 21(4), 399-411. https://doi.org/10.7202/019163ar
Résumé de l'article

Des études portant sur l'épuration des eaux usées domestiques ont été menées sur une filière de trois bassins en série de lagunage à microphytes à la station expérimentale de l'Institut International d'Ingénierie de l'Eau et de l'Environnement (2IE). Cette filière comporte : un bassin anaérobie (BA), un bassin facultatif (BF) et un bassin de maturation (BM). Des mesures d'indicateurs de qualité ont été effectuées pendant dix mois sur des échantillons prélevés suivant une fréquence bihebdomadaire pour certains et hebdomadaire pour d'autres. Les rendements épuratoires moyens atteignent $66 \%$ pour les MES, $81 \%$ pour la DCO et $87 \%$ pour la $\mathrm{DBO}_{5}$. Le résiduel de MES de l'effluent traité répond, dans $76 \%$ des mesures, aux normes de rejet recommandées par la directive de l'Union Européenne $\left(<150 \mathrm{mg} \mathrm{L}^{-1}\right)$. L'élimination du phosphore est faible et instable avec un rendement moyen de $17,2 \%$ pour le phosphore total et $19,2 \%$ pour les ortho-phosphates. La réduction de la pollution azotée atteint en moyenne $76 \%$ en NTK et $38,4 \%$ en azote ammoniacal. De manière globale, les teneurs résiduelles en azote et en phosphore restent assez élevées par rapport à la limite tolérable pour un rejet d'effluent dans un écosystème sensible à l'eutrophisation. L'abattement de la pollution bactérienne est important allant jusqu'à 4,9 ulog pour les coliformes fécaux; mais la teneur résiduelle moyenne $\left(5,4 \times 10^{3}\right.$ unités formant des colonies (ufc)/100 mL) reste tout de même supérieure à la directive OMS ( $\leqslant 1000 \mathrm{ufc} / 100 \mathrm{~mL}$ ) pour une réutilisation non restrictive en irrigation. Concernant la charge parasitaire, les études ont montré que la filière assure une élimination totale $(100 \%)$ des oeufs d'helminthes et des kystes de protozoaires. 


\section{PERFORMANCES ÉPURATOIRES D'UNE FILIÈRE DE TROIS BASSINS EN SÉRIE DE LAGUNAGE À MICROPHYTES SOUS CLIMAT SAHÉLIEN : CAS DE LA STATION DE TRAITEMENT DES EAUX USÉES DE 21E (GROUPE EIER-ETSHER)}

Performance of a series of three wastewater stabilisation ponds in Sahelian climate: Case study of 21E (EIER-ETSHER) wastewater treatment plant

Amadou Hama Maiga ${ }^{1 *}$, Yacouba Konate ${ }^{1}{\text { Joseph } \text { Wethe }^{1} \text {, Kokou Denyigba }}^{1}$, Denis Zoungrana ${ }^{1}$, Lassana Togola ${ }^{1}$ ${ }^{1}$ Institut International de l'Ingénierie de l'Eau et de l'Environnement (2IE)/Groupe EIER-ETSHER 01 BP 594, Ouagadougou 01, Burkina Faso

Reçu le 22 novembre 2006, accepté le 4 décembre 2007

\section{RÉSUMÉ}

Des études portant sur l'épuration des eaux usées domestiques ontété menées sur unefilière de trois bassins en série de lagunageà microphytes àla station expérimentale del'Institut International d'Ingénierie de l'Eau et de l'Environnement (2IE). Cette filière comporte : un bassin anaérobie (BA), un bassin facultatif $(\mathrm{BF})$ et un bassin de maturation (BM). Des mesures d'indicateurs de qualité ont été effectuées pendant dix mois sur des échantillons prélevés suivant une fréquence bihebdomadaire pour certains et hebdomadaire pour d'autres. Les rendements épuratoires moyens atteignent $66 \%$ pour les MES, $81 \%$ pour la DCO et $87 \%$ pour la $\mathrm{DBO}_{5}$. Le résiduel de MES de l'effluent traité répond, dans $76 \%$ des mesures, aux normes de rejet recommandées par la directive de l'Union Européenne $\left(<150 \mathrm{mg} \mathrm{L}^{-1}\right)$. L'élimination du phosphore est faible et instable avec un rendement moyen de $17,2 \%$ pour le phosphore total et $19,2 \%$ pour les ortho-phosphates. La réduction de la pollution azotée atteint en moyenne $76 \%$ en NTK et $38,4 \%$ en azote ammoniacal. De manière globale, les teneurs résiduelles en azote et en phosphore restent assez 
élevées par rapport à la limite tolérable pour un rejet d'effluent dans un écosystème sensible à l'eutrophisation. L'abattement de la pollution bactérienne est important allant jusqu'à 4,9 ulog pour les coliformes fécaux; mais la teneur résiduelle moyenne $\left(5,4 \times 10^{3}\right.$ unités formant des colonies $\left.(\mathrm{ufc}) / 100 \mathrm{~mL}\right)$ reste tout de même supérieure à la directive OMS ( $\leq 1000 \mathrm{ufc} / 100 \mathrm{~mL})$ pour une réutilisation non restrictive en irrigation. Concernant la charge parasitaire, les études ont montré que la filière assure une élimination totale $(100 \%)$ des œufs d'helminthes et des kystes de protozoaires.

\section{Mots clés : Traitement, eaux usées, lagunage à microphytes, climat sabélien.}

\section{ABSTRACT}

Waste stabilization ponds (WSP) are considered to be one of the main methods for municipal wastewater because of their advantages over other methods (conventional treatment) relating to treatment effectiveness, system flexibility and simplicity, and cost (ARTHUR, 1983; MARA, 1976; MARA and PEARSON, 1986; SILVA, 1982). Moreover, it has long been accepted that tropical climates provide an ideal environment for the natural treatment of sewage in ponds.

Since 1989, the 2IE (International Institute for water and Environmental Engineering) wastewater treatment plant in the city of Ouagadougou (Burkina Faso) has been investigating the performance of pilot-scale systems in order to establish regional design parameters and removal efficiencies. Several experiments have been carried out on pond series with different configurations and operational characteristics aiming to improve the design, operation and performance of waste stabilization ponds in Sahelian climate conditions. The purpose of the present study was to evaluate the efficiency of a new series of three stabilization ponds.

The series was composed by one anaerobic pond (AP), one facultative pond (FP) followed by one maturation pond (MP). The characteristics (depth, area, volume and hydraulic retention time) were respectively $2.6 \mathrm{~m}, 69.7 \mathrm{~m}^{2}, 181.2 \mathrm{~m}^{3}$, and three days for the AP, $1.4 \mathrm{~m}, 415.3 \mathrm{~m}^{2}$ and $581.4 \mathrm{~m}^{3}$ and 9.5 days for the FP, $0.9 \mathrm{~m}, 336.9 \mathrm{~m}^{2}, 303.2 \mathrm{~m}^{3}$ and 5.5 days for the MP. The ponds were constructed with concrete to ensure water tightness. Field studies indicated a wide fluctuation in the biochemical oxygen demand $\left(\mathrm{BOD}_{5}\right)$ loading applied. The average volumetric organic load ranged from 104 to $225 \mathrm{~g}$ $\mathrm{BOD}_{5} \mathrm{~m}^{-3} \mathrm{j}^{-1}$ with an average load of $105.2 \mathrm{~g} \mathrm{BOD}_{5} \mathrm{~m}^{-3} \mathrm{j}^{-1}$ for the AP, the average surface $\mathrm{BOD}_{5}$ load on the $\mathrm{FP}$ was $117 \mathrm{~kg}$ $\mathrm{BOD}_{5} / \mathrm{ha} / \mathrm{j}$.
The series was monitored for ten months from October 2004 to July 2005. The assessment involved an intensive program of sampling and analysis. The composite samples (obtained daily by mixing equal volumes of grab samples collected) were taken from the influent and effluent of each pond twice per week. Global physico-chemical parameters $\left(\mathrm{T}^{\circ}, \mathrm{pH}\right.$, Dissolved Oxygen (DO), conductivity) were measured in situ. Chemical Oxygen Demand (COD), Biological Oxygen Demand (BOD) were analyzed according to standard methods. Suspended solids (SS) were determined gravimetrically after filtration through Whatman GF/C glass fiber filters and drying at $105^{\circ} \mathrm{C}$. Total Kjeldahl Nitrogen (TKN) and ammonia nitrogen (AN) were analyzed according to AFNOR methods. Nitrate nitrogen (NT), total phosphorus (TP) and orthophosphate (OP) were determined by HACH methods. Faecal coliforms (FC) were counted by layer technique using the chromocult coliform agar, helminth eggs and protozoan cysts were determined by sodium acetate formalin (SAF) adapted method.

Results showed during the study period average removal rates of $66 \%$ for SS, $87 \%$ for filtered $\mathrm{BOD}_{5}$, and $81 \%$ for $\mathrm{COD}$. For $\mathrm{COD}$ and $\mathrm{BOD}_{5}$, removal performances were relatively constant over the study period with ranges of $(60,9 \%-96,1 \%)$ and $(65,6-6,8 \%)$, respectively. In $83.8 \%$ of filtered $\mathrm{BOD}_{5}$ measures $(\mathrm{n}=68)$ on the final effluent, $\mathrm{BOD}_{5}$ removal efficiency were higher than $80 \%$. However, for SS removal efficiency, it indicated a wide fluctuation. The same fluctuation was observed on SS concentration on the final effluent, with a variation coefficient of $34.3 \%$. Effluent SS concentrations from the series varied between a minimum of $4 \mathrm{mg} / \mathrm{L}$ and a maximum of $326 \mathrm{mg} / \mathrm{L}$ with average of $110 \mathrm{mg} /$ L. The higher values of SS were observed during the warm period (March-April) corresponding to the abundance of algal proliferation in facultative and maturation ponds. In this period, effluent SS concentrations were higher than the influent ones, leading to negative efficiencies for these ponds. Despite this disturbance due to algal proliferation, the final effluent quality met the European Union (EU) quality requirements, i.e suspended solids concentration $<150 \mathrm{mg}$ per litre. This standard was achieved in $76 \%$ of SS measures $(n=75)$ during the study period.

The capability of the system for phosphorus removal was weak and variable, with average removals around $17 \%$ and $19 \%$ respectively for $\mathrm{TP}$ and OP removals. The mean residual concentrations of TP and OP in the final effluent were respectively $12.5 \mathrm{mg} / \mathrm{L}$ and $4.6 \mathrm{mg} / \mathrm{L}$, values higher than the maximum concentration of $0.1 \mathrm{mg} / \mathrm{L}$ PT for the discharge of effluent in water sensitive to eutrophication (AYERS and WESTCOT,1985; WHO, 1989). Average TKN removal was $76 \%$. Global efficiencies of the three-pond series for nutrients (nitrogen and phosphorus) reduction were weak and the effluent concentration of these nutrients did not satisfy the quality recommended to discharge in water sensitive to 
eutrophication. However, they could be useful as fertilizer for reuse for irrigation.

With a total hydraulic retention time (HRT) of 18 days, the removal of faecal coliforms $(\mathrm{fc})$ reached a maximum removal of $4.9 \mathrm{log}$ units. The residuals $\mathrm{fc}$ concentration in the final effluent ranged between $1 \times 10^{3} \mathrm{fc} / 100 \mathrm{~mL}$ to $3.4 \times 10^{5} \mathrm{fc} /$ $100 \mathrm{~mL}$, with an average of $5.4 \times 10^{3} \mathrm{fc} / 100 \mathrm{~mL}$. According to the WHO recommendation (1989) for reuse in agriculture, the effluent did not meet the WHO guideline $(\leq 1000 \mathrm{fc}$ per $100 \mathrm{~mL}$ ) for unrestricted irrigation. In spite of an average HRT of 18 days, the results showed that the fc removal for the WSP system (AP + FP + MP) in a Sahelian climate did not meet the WHO standard for faecal coliform reduction. The performance results of this study were, however, slightly better than those obtained by MADERA et al. (2002) on a similar study in wet tropical climate (in Colombia). Concerning helminths eggs and protozoan cysts, different parasites were identified in the raw wastewater such as: eggs of ankylostoms ranging from 0 to 4 eggs/litre, ascaris eggs 0 - 10 eggs/litre, trichuris eggs 0 - 1 egg/litre, and larval stages of anguillula $0-5$ eggs/litre. Two protozoan cysts were identified in the raw wastewater: Entamoeba coli ranged 6-272 cysts/litre and entamoeba histolitica $2-55$ cysts/litre. Concerning helminths eggs and protozoan cysts removals, they were all completely (100\%) removed in the final effluent of the series after 18 days HRT. Helminth eggs and protozoan cysts removal was very good. Most were removed in the anaerobic and facultative ponds, and the WHO guideline values for crop irrigation (both restricted and unrestricted) of $<1$ egg/litre were achieved after the two first ponds $(\mathrm{AP}+\mathrm{FP})$. Regarding sanitary concerns during the ten-month study period, the final effluent of the three ponds series correspond to B category effluent (WHO,1989) which could be reused for irrigation of cereal crops, industrial crops, fodder crops, pasture, and trees.

\section{Key words: Treatment, wastewater, waste stabilization ponds, Sahelian climate}

\section{INTRODUCTION}

Diverses études réalisées en 1993 sur des systèmes de traitement des eaux usées ont révéléqueles technologiesintensives basées sur le système de boues activées, qui représentaient $75 \%$ des stations construites en Afrique subsaharienne, sont inadaptées. Cette insuffisance est due à plusieurs facteurs tels le coût d'exploitation élevé, la non-disponibilité des pièces de rechange, le manque d'expérience et le faible niveau de formation du personnel en charge de la gestion de ces systèmes $(\mathrm{CIEH}$, 1993). Le principal défi consiste à développer des options de traitement qui soient à la fois efficaces et techniquement adaptées aux conditions environnementales et financières des pays africains. De façon évidente, les technologies naturelles d'épuration extensive peu onéreuses constituent des options de traitement adaptées, en particulier devant l'intérêt croissant pour la réutilisation de ces eaux usées en agriculture urbaine dans les pays sahéliens.

On note depuis les années 1980 la concentration, dans certains pays de la sous-région Afrique de l'Ouest, de quelques stations de lagunage à microphytes. Dans bien des cas, les conditions de fonctionnement de ces stations se sont révélées différentes de celles qui ont présidé à leur conception. Par conséquent, il devient ainsi difficile de tirer des enseignements valables sur leur performance. Les modèles de dimensionnement des bassins de lagunage étant calés dans des conditions différentes, une étude dans les conditions climatiques sahéliennes se justifie.

Le Groupe EIER-ETSHER (devenu Institut International d'Ingénierie de l'Eau et de l'Environnement (2IE), sis à Ouagadougou au Burkina Faso), a initié depuis 1989 des recherches sur le fonctionnement des systèmes de lagunage sous climat sahélien. Les premières études sur les performances épuratoires (GUÈNE, 1989; KLUTSÉ, 1995; RADIN, 1990) ont été effectuées sur des filières à deux bassins en série composées chacune d'un bassin facultatif et d'un bassin de maturation et des filières à lit bactérien. Cet article rapporte les résultats d'une année d'étude menée de 2004 à 2005 sur une filière de trois bassins en série (Bassin Anaérobie, Bassin Facultatif et Bassin de Maturation).

\section{MATÉRIELS ET MÉTHODES}

\subsection{Description du site}

L'étude a été réalisée à la station pilote expérimentale (220 Équivalent-Habitant) du 2IE (Groupe EIER-ETSHER) à Ouagadougou (Figure 1). Le fonctionnement de cette station est régi par le climat soudano-sahélien de Ouagadougou marqué par une longue période sèche, d'octobre à mai (soit huit mois), avec un ensoleillement important en durée (2500 heures d'ensoleillement par an) et en intensité (19,5 - 22,7 MJ/m²/J) et des pertes en eau par évaporation variant de $8 \mathrm{~mm} / \mathrm{j}$ en moyenne. La sollicitation climatique évaporatoire est négligeable par rapport à la configuration géométrique des bassins et les pertes en eau ne présentent pas d'impact significatif sur le pouvoir épuratoire des systèmes de lagunage. La saison pluvieuse est caractérisée par des pluies irrégulières sporadiques. Construite en 1989, et après une dizaine d'années de fonctionnement, cette station a été réaménagée avec une réorganisation des 


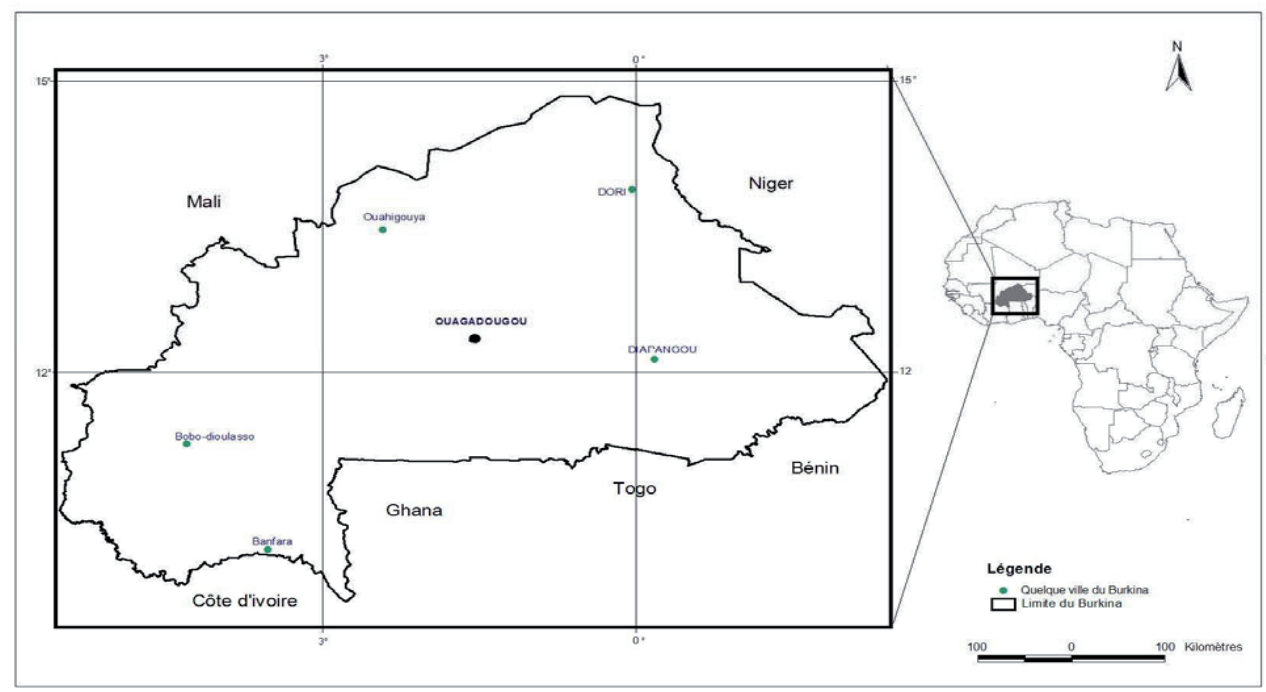

Figure 1. Situation géographique du cadre de l'étude. Geographic location of the 2IE treatment plant.

filières de traitement. Cette réorganisation a abouti à un nouvel agencement des bassins de lagunage. L'innovation résulte surtout de l'introduction d'un bassin anaérobie pour servir de traitement primaire des eaux usées brutes en remplacement du décanteur primaire dont les rendements obtenus dans les études antérieures étaient trop bas avec, par exemple, un abattement moyen de la Demande Chimique en Oxygène (DCO) inférieur à $20 \%$. La station de lagunage du 2IE est ainsi composée de cinq filières (Figure 2) :

- Filière $\mathrm{n}^{\circ} 1$ : composée en série d'un bassin anaérobie (BA), un bassin facultatif (BF) et un bassin de maturation (BM).

- Filière $\mathrm{n}^{\circ} 2$ : composée d'un décanteur primaire, un bassin facultatif, et un bassin de maturation.

- Filière $n^{\circ} 3$ : un décanteur primaire, un lit bactérien rectangulaire, un décanteur secondaire, et un bassin de maturation.

- Filière ${ }^{\circ} 4$ : un décanteur primaire, un lit bactérien circulaire, un décanteur secondaire, et un bassin de maturation.

- Filière $\mathrm{n}^{\circ} 5$ : un décanteur primaire, des bassins à macrophytes de pistia stratriote.

Les caractéristiques des bassins de la filière 1, cadre de l'étude, sont consignées dans le tableau 1.

La station est alimentée par les eaux usées produites sur le campus du 2IE (en sont exclues les eaux usées issues des laboratoires). Le débit journalier admis à l'entrée de la filière 1 est en moyenne de $55 \mathrm{~m}^{3} / \mathrm{j}$. Au cours de l'étude, les charges organiques volumiques admises (mesurées en demande biologique en oxygène après cinq jours $\left.\left(\mathrm{DBO}_{5}\right)\right)$ ont varié de 104 à $225 \mathrm{~g} \mathrm{DBO}_{5} \mathrm{~m}^{-3} \mathrm{j}^{-1}$ avec une charge moyenne de $105,2 \mathrm{~g}$ $\mathrm{DBO}_{5} \mathrm{~m}^{-3} \mathrm{j}^{-1}$. Cette variation de charge est liée aux activités fluctuantes de consommation d'eau du campus, marquées par des périodes de départ des élèves pour congés ou vacances et d'arrivées multiples de stagiaires pour des formations continues. Sur le bassin facultatif, les charges surfaciques admises à partir de l'effluent issu du bassin anaérobie vont de 117 à $419 \mathrm{~kg}$ $\mathrm{DBO}_{5} / \mathrm{ha} / \mathrm{j}$, soit en moyenne $288,3 \mathrm{~kg} \mathrm{DBO}_{5} / \mathrm{ha} / \mathrm{j}$.

\subsection{Méthodologie d'échantillonnage et de mesure}

La méthode d'échantillonnage adoptée est de type composite avec des mesures bihebdomadaires sur une période de dix mois (octobre 2004 - juillet 2005). Les points de prélèvement ont été effectués à l'entrée et à la sortie de chaque bassin, ce qui a permis de suivre la part d'épuration de chaque bassin, et la capacité épuratoire de l'ensemble de la filière. Les paramètres physico-chimiques globaux (Température $\left(\mathrm{T}^{\circ}\right), \mathrm{pH}$, Oxygène $\left(\mathrm{O}_{2}\right)$ dissous, conductivité électrique) ont été mesurés in situ au moyen d'une sonde multiparamètres (multiline P4). Les mesures ont été faites sur des échantillons ponctuels prélevés trois fois par jour (matin entre $7 \mathrm{~h}-8 \mathrm{~h}$, midi entre $12 \mathrm{~h}-13 \mathrm{~h}$ et l'après-midi entre $17 \mathrm{~h}-18 \mathrm{~h}$ ). Les paramètres physicochimiques et biologiques (la $\mathrm{DBO}_{5}$, la $\mathrm{DCO}$, les matières en suspension (MES), l'azote, le phosphore) ont été mesurés sur des échantillons composites journaliers constitués en proportions égales d'échantillons ponctuels prélevés à l'entrée et à la sortie du bassin. Les fréquences d'analyse ont varié d'un paramètre à un autre, compte tenu de la disponibilité du matériel d'analyse. Les analyses bactériennes (coliformes fécaux) et parasitologiques (kystes de protozoaires et œufs d'helminthes) ont également été effectuées suivant les méthodes citées dans le tableau 2. 


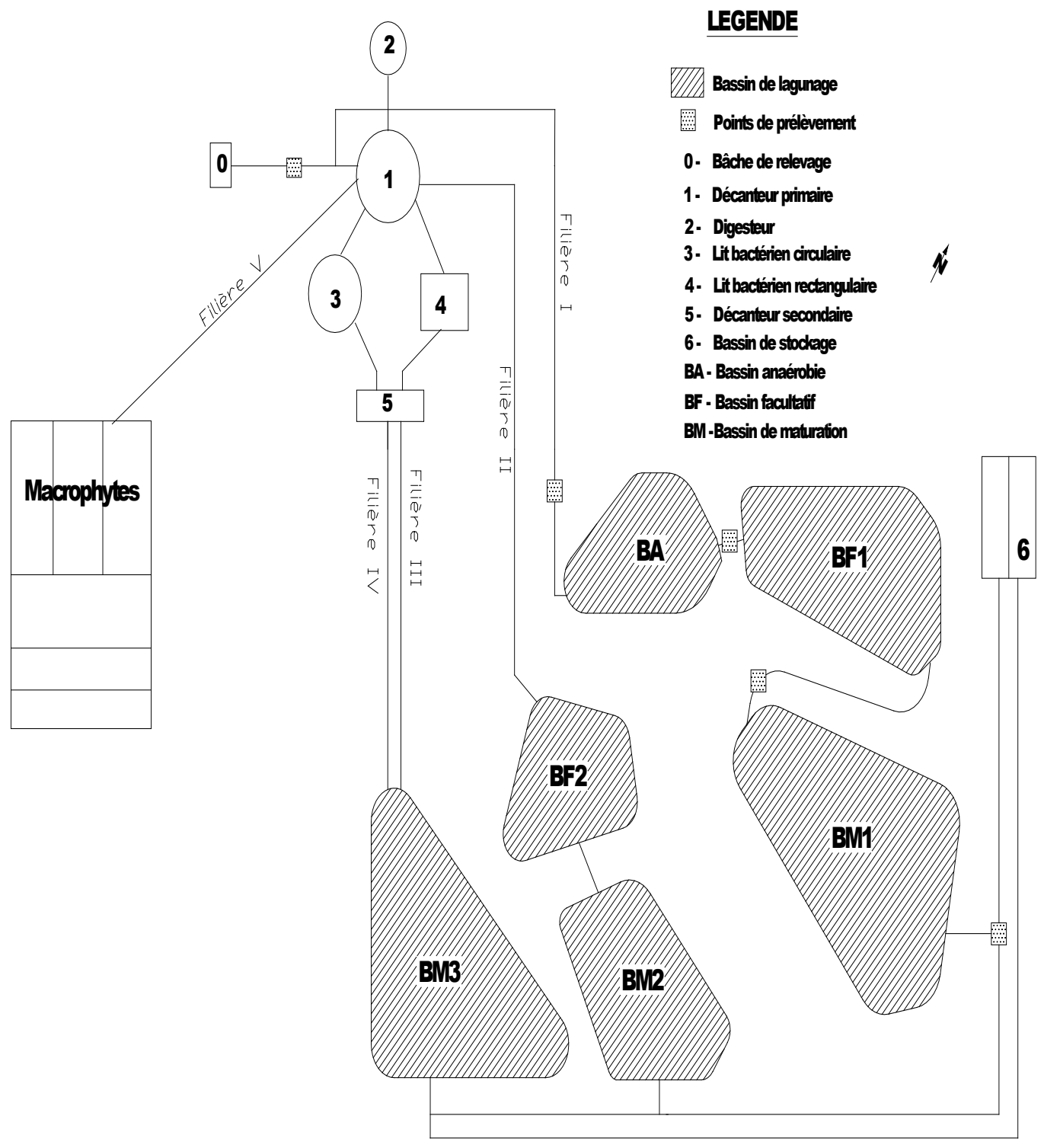

Figure 2. Schéma général des filières de lagunage de la station expérimentale pilote de 2IE. Schematic representation of the pilot-scale wastewater stabilization ponds at $2 I E$.

Tableau 1. Caractéristiques des bassins de lagunage de la filière étudiée.

Table1: $\quad$ Characteristics of the series of ponds studied.

\begin{tabular}{lcccc}
\hline Bassin & Profondeur $(\mathrm{m})$ & Surface $\left(\mathrm{m}^{2}\right)$ & Volume $\left(\mathrm{m}^{3}\right)$ & $\begin{array}{c}\text { Temps de rétention } \\
\text { hydraulique (jours) }\end{array}$ \\
\hline Bassin anaérobie & 2,6 & 69,7 & 181,2 & 3 \\
$\begin{array}{l}\text { Bassin facultatif } \\
\begin{array}{l}\text { Bassin de } \\
\text { maturation }\end{array}\end{array}$ & 1,4 & 415,3 & 581,4 & 9,5 \\
\hline
\end{tabular}


Tableau 2. Paramètres analysés et méthodes d'analyse.

Table 2. Parameters and methods used in analysis.

\begin{tabular}{|c|c|c|}
\hline Paramètres & Unités & Méthodes d'analyse \\
\hline Matière En Suspension (MES) & $(\mathrm{mg} / \mathrm{L})$ & AFNOR T90-105 \\
\hline Demande Chimique en Oxygène (DCO) & $\left(\mathrm{mgO}_{2} / \mathrm{L}\right)$ & Standard methods. $18^{\mathrm{e}}$ édition \\
\hline Demande Biologique Oxygène $\left(\mathrm{DBO}_{5}\right)$ & $\left(\mathrm{mgO}_{2} / \mathrm{L}\right)$ & Standard methods. $18^{e}$ édition \\
\hline Sulfate & $(\mathrm{mg} / \mathrm{L})$ & HACH 8051* méthode 8051 Sulfaver 4 \\
\hline Azote Total Kjeldahl (NTK) & $(\mathrm{mgN} / \mathrm{L})$ & AFNOR T90-110 \\
\hline Azote Ammoniacal N-NH${ }_{4}^{+}$ & $(\mathrm{mgN} / \mathrm{L})$ & AFNOR T90-015 \\
\hline Azote Nitrique $\mathrm{N}^{-\mathrm{NO}_{3}^{-}}$ & $(\mathrm{mgN} / \mathrm{L})$ & HACH 8039 méthode de réduction au cadmium \\
\hline Phosphore Total PT & $(\mathrm{mgP} / \mathrm{L})$ & HACH 8190* méthode de digestion au persulfate acide \\
\hline Ortho-phosphate $\mathrm{P}_{-} \mathrm{PO}_{4}^{3-}$ & $(\mathrm{mgP} / \mathrm{L})$ & HACH 8114* méthode au molybdovadate \\
\hline Coliformes Fécaux & (ucf/100 mL) & $\begin{array}{l}\text { Etalement sur gélose spécifique Chromocult pour } \\
\text { coliforme }\end{array}$ \\
\hline Cufs d'helminthes & ( (Euf/L) & $\begin{array}{l}\text { Méthode SAF adaptée suivi de l'identification et comptage } \\
\text { au microscope }\end{array}$ \\
\hline Kystes de protozoaires & (Kyste/L) & $\begin{array}{l}\text { Méthode SAF adaptée suivi de l'identification et comptage } \\
\text { au microscope }\end{array}$ \\
\hline
\end{tabular}

\section{RÉSULTATS ET DISCUSSION}

\subsection{Caractéristiques de l'influent brut}

Les caractéristiques de l'influent brut admis en tête de la filière sont présentées dans le tableau 3.

Les valeurs de la $\mathrm{DCO}$ et de la $\mathrm{DBO}_{5}$ restent dans la gamme des valeurs de référence pour les eaux usées d'origine domestique avec un rapport moyen de $\mathrm{DCO} / \mathrm{DBO}_{5}$ de 1,37 . Ce rapport étant inférieur à 2 permet de conclure qu'il s'agit d'un influent brut facilement biodégradable (METCALF et EDDY (1991); GSL MEMOTEC $\left.\mathrm{N}^{\circ} 19\right)$. Avec des coefficients de variation pour la DCO, la $\mathrm{DBO}_{5}$, et les MES respectivement de 31,4\%, $37,5 \%$, et $38 \%$, ce qui révèle une situation de forte variabilité de la qualité de l'influent admis en tête de la station. Les valeurs de $\mathrm{pH}$ évoluent dans une gamme de 5,63-8,68, ce qui est de nature à favoriser le développement bactérien nécessaire à la dégradation biologique des polluants organiques.

Les charges organiques volumiques apportées par les eaux brutes sont conformes aux données de littérature qui rapportent des charges admissibles de 100 à $400 \mathrm{~g} \mathrm{DBO} / \mathrm{m}^{3} / \mathrm{j}$ sur un bassin anaérobie (ARTHUR, 1983). Au niveau du basin facultatif, les charges surfaciques admises restent pratiquement dans les limites conventionnelles admissibles recommandées pour ce type de bassin, soit $100-400 \mathrm{~kg} \mathrm{DBO}_{5} / \mathrm{ha} / \mathrm{j}$ (ABIS, 2002), exceptés les mois de janvier et février où l'on a observé un dépassement de 4,75\% par rapport à la charge maximale limite recommandée qui est de $400 \mathrm{~kg} \mathrm{DBO} / \mathrm{ha} / \mathrm{j}$.

\subsection{Rendements épuratoires}

\subsection{1 Élimination de la charge organique}

Les figures 3, 4 et 5 présentent respectivement l'évolution des teneurs et des rendements épuratoires pour les MES, la $\mathrm{DBO}_{5}$ et la DCO de l'influent brut à l'entrée de la filière et de l'effluent épuré à la sortie du bassin de maturation.

Les rendements moyens d'abattement de la pollution sur la période de suivi sont de $66 \%$ pour les MES, $87 \%$ pour la $\mathrm{DBO}_{5}$ filtrée et $81 \%$ pour la DCO. Pour la DCO et la $\mathrm{DBO}_{5}$, les rendements d'épuration ont été relativement constants dans le temps, restant respectivement dans les intervalles de $(60,9 \%-96,1 \%]$ et $(65,6-96,8 \%)$. L'écarttype et le coefficient de variation des valeurs mesurées sont respectivement de 6,2 et $7,6 \%$ pour la DCO, 7,5 et 8,6\% pour la $\mathrm{DBO}_{5}$ filtrée. Cette constance en matière de rendement épuratoire en $\mathrm{DCO}$ et $\mathrm{DBO}_{5}$ filtrée confirme d'avantage la forte robustesse des systèmes naturels d'épuration des eaux usées, qui peuvent supporter des variations de charge organique tant que celle- ci reste dans la gamme de charge admissible. Pour la $\mathrm{DBO}_{5}$ filtrée de l'effluent traité, les performances épuratoires ont été supérieures à $80 \%$ dans 83,8\% des mesures $(n=68)$. Le rendement moyen de $87 \%$ d'abattement 
Tableau 3. Caractéristiques des eaux usées brutes.

Table 3. Raw sewage characteristics.

\begin{tabular}{lcccc}
\hline Paramètres & Moyenne & Intervalle & Écart-type & Nombre d'échantillons \\
\hline Température ${ }^{\circ} \mathrm{C}$ & $\mathbf{2 9 , 6}$ & $18,2-35,2$ & 2,69 & 225 \\
$\mathrm{pH}$ & $\mathbf{7 , 4 7}$ & $5,63-8,68$ & 0,51 & 225 \\
$\mathrm{O}_{2}$ dissous & $\mathbf{0 , 8 9}$ & $0.21-6.00$ & 0,47 & 225 \\
Conductivité $\mu \mathrm{S} / \mathrm{cm}$ & $\mathbf{6 5 5}$ & $117-944$ & 121 & 225 \\
$\mathrm{DCO} \mathrm{mg} / \mathrm{L}$ & $\mathbf{6 5 2}$ & $295-1026$ & 204 & 75 \\
$\mathrm{DBO}_{5} \mathrm{mg} / \mathrm{L}$ & $\mathbf{4 8 9}$ & $160-800$ & 185 & 67 \\
$\mathrm{MES} \mathrm{mg/L}$ & $\mathbf{3 2 0}$ & $112-658$ & 121 & 75 \\
$\mathrm{Phosphore} \mathrm{total} \mathrm{mg/L}_{\text {Orthophosphate mg/L }}^{\mathbf{5 , 7 9}}$ & $4,10-68,00$ & 11,81 & 75 \\
$\mathrm{NTK} \mathrm{mg/L}$ & $\mathbf{5 , 1 3}$ & $2,61-9,38$ & 1,65 & 75 \\
$\mathrm{~N}-\mathrm{NH}_{4}^{+} \mathrm{mg} / \mathrm{L}$ & $\mathbf{2 0 9 . 3}$ & $44,80-425,60$ & 80,69 & 19 \\
$\mathrm{~N}-\mathrm{NO}_{3}^{-} \mathrm{mg} / \mathrm{L}$ & $\mathbf{4 7 , 3}$ & $17,09-86,43$ & 12,21 & 75 \\
$\mathrm{CF}$ ulog & $\mathbf{4 , 0 3}$ & $1,10-13,10$ & 2,29 & 73 \\
\hline
\end{tabular}

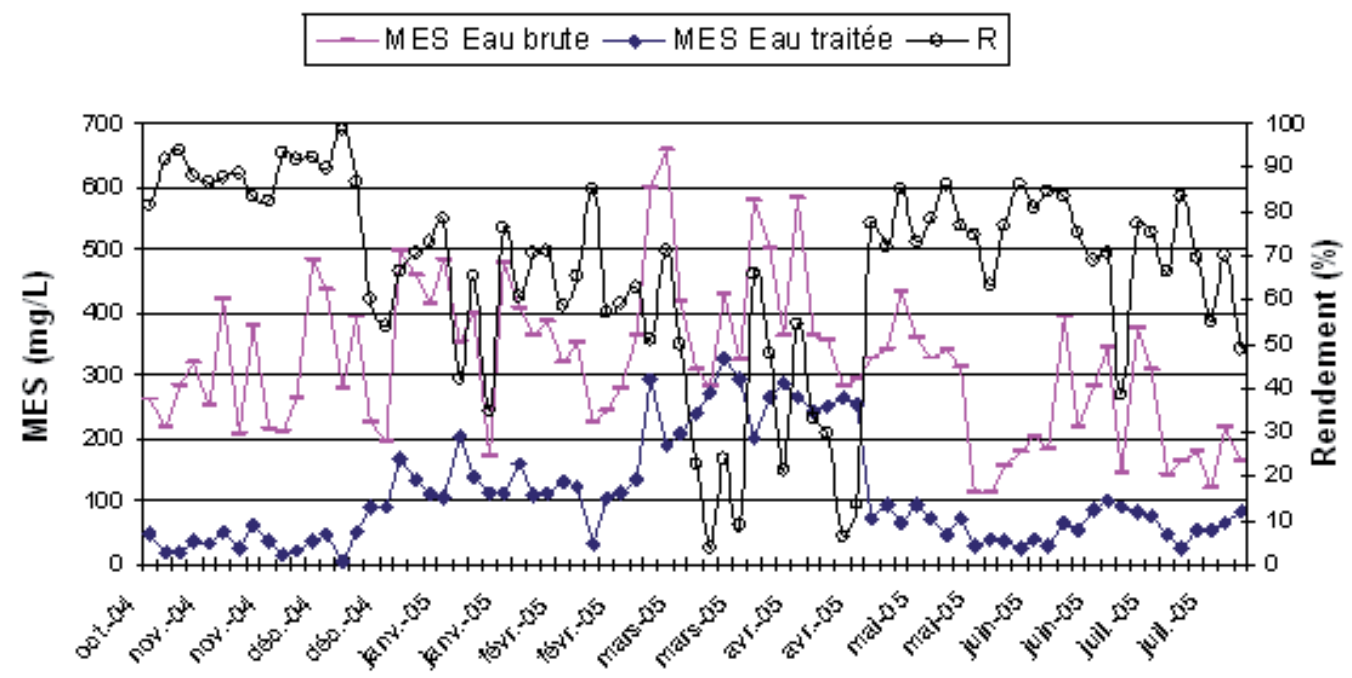

Figure 3. Variation des concentrations en MES et de son taux d'abattement à travers le système. Suspended solids (SS) variations and removal efficiency throughout the system.

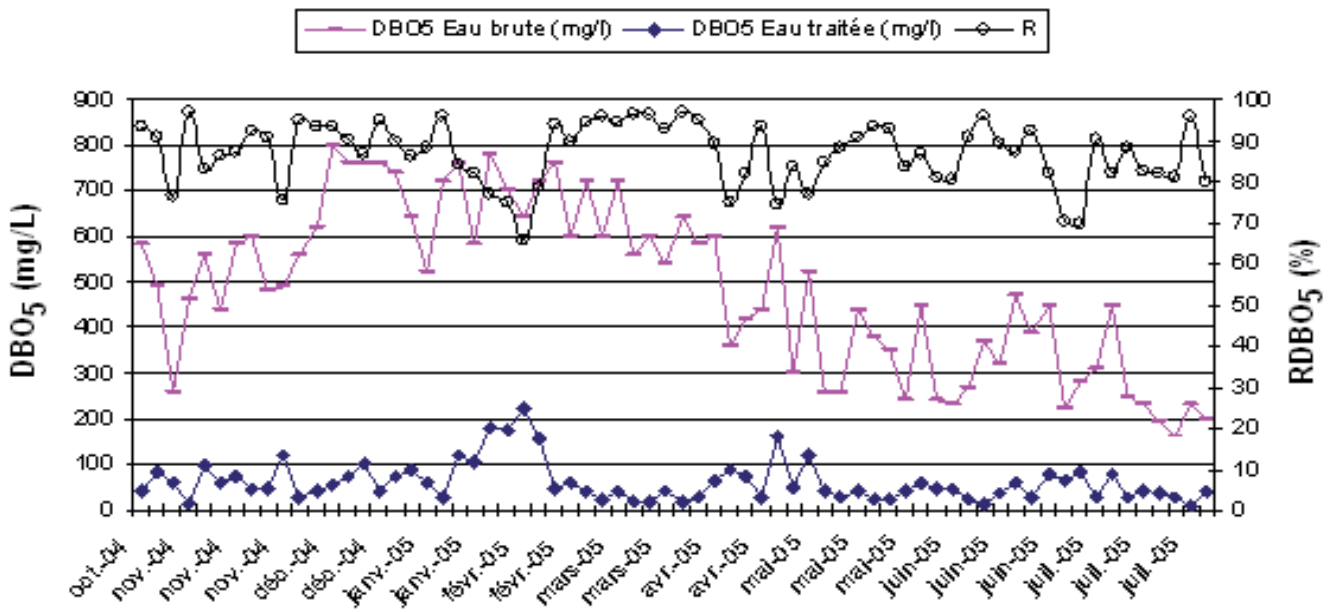

Figure 4. Variation de la $\mathrm{DBO} 5$ et de son taux d'abattement à travers le système. $B O D_{5}$ variations and removal efficiency throughout the system. 


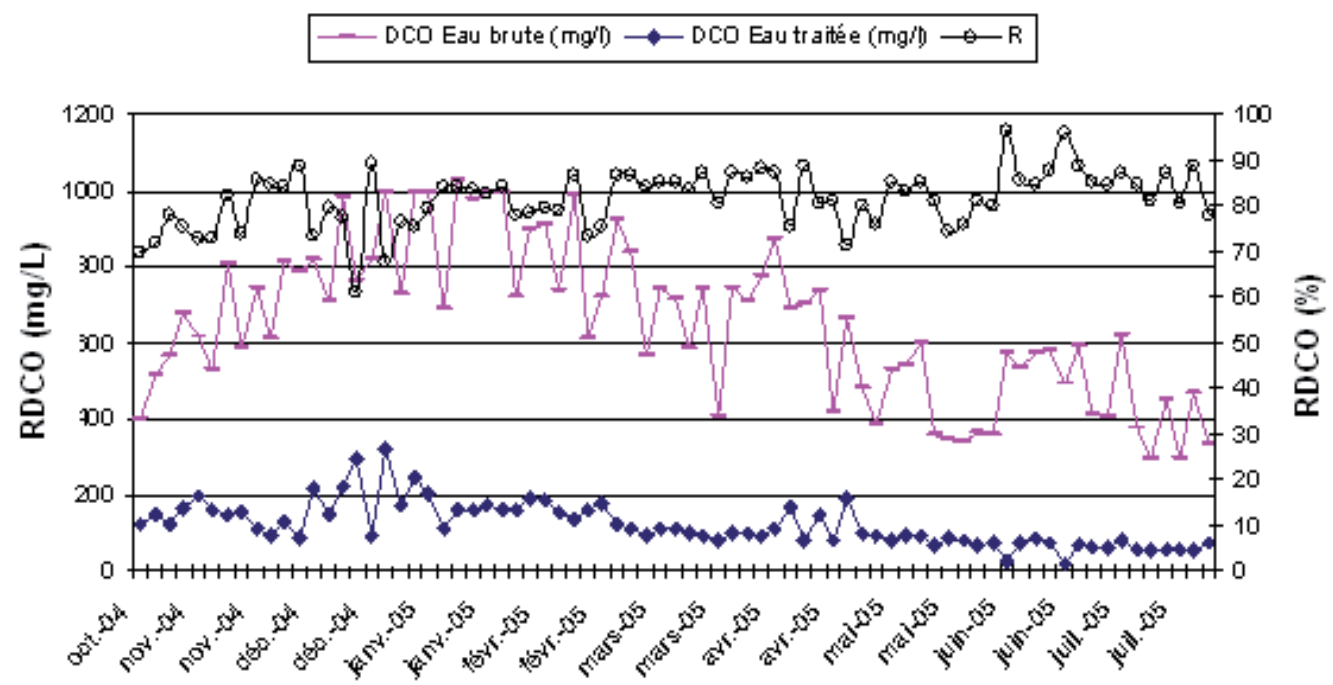

Figure 5. Variation de la DCO et de son taux d'abattement à travers le système . $C O D$ variation and removal efficiency throughout the system.

est un abattement satisfaisant relativement de même ordre de grandeur que le rendement prévu (>90\%) pour une série convenablement dimensionnée de lagunage à microphyte (MARA et PEARSON, 1998). L'efficacité du bassin anaérobie comme traitement primaire dans la série s'est traduite par une réduction significative de la pollution organique $\left(\mathrm{DBO}_{5}, \mathrm{DCO}\right.$, MES) avec des performances intrinsèques respectivement de $49,7 \%, 40,2 \%$ et $69,5 \%$. Signalons que dans les études antérieures, le traitement primaire dans le décanteur primaire n'a pas permis d'atteindre un niveau d'abattement satisfaisant : RADIN (1990) rapporte des rendements faibles de $38 \%$ pour les MES, et seulement $11 \%$ pour la DCO, KLUTSÉ (1995) rapporte un rendement de $22 \%$ en $\mathrm{DBO}_{5}$ sur une performance globale de $76 \%$ pour la succession ancienne décanteur primaire $+\mathrm{BF}+\mathrm{BM}$ avec un temps de rétention hydraulique (TRH) de 16,2 jours. La raison fondamentale $\mathrm{du}$ faible rendement au niveau du décanteur primaire statique serait un défaut de soutirage régulier de boues. Celles-ci sont entraînées avec des charges trop élevées (1500 kg/ha/jour selon KLUSTÉ, 1995) dans le bassin facultatif qui fonctionnait ainsi en surcharge. Avec l'introduction du bassin anaérobie, l'impact positif relevé est sa capacité de laminage des pics de pollution protégeant ainsi le bassin facultatif contre les à coups de charge.

Pour l'élimination des MES, le système montre de grandes fluctuations pour l'abattement global à la sortie de la station (Figure 3). À la sortie du bassin de maturation, on relève une variation importante des concentrations en MES avec un coefficient de variation de 34,3\% pour une teneur moyenne de $110 \mathrm{mg} / \mathrm{L}$. Le minimum observé est de $4 \mathrm{mg} / \mathrm{L}$ et le maximum de $326 \mathrm{mg} / \mathrm{L}$. Les fortes valeurs en MES de l'effluent traité ont été observées surtout en période de chaleur (mars-avril) correspondant à la période de développement abondant du phytoplancton. En effet, la période de bloom algal est caractérisée par une pollution organique de néoformation qui gêne souvent l'abattement de la pollution et peut empêcher l'atteinte des objectifs de qualité en ce qui a trait à la concentration résiduelle en MES. Ce phénomène apparaît surtout dans les bassins facultatifs et de maturation où l'on a observé par moment des teneurs en $\mathrm{DCO}, \mathrm{DBO}_{5}$ et MES supérieures pour l'effluent en sortie de ces bassins par rapport à celles des flux entrants. La contribution de la charge algale de néoformation dans la $\mathrm{DBO}_{5}$ totale est contenue dans la $\mathrm{DBO}_{5}$ particulaire qui correspond à la différence entre la $\mathrm{DBO}_{5}$ totale et la $\mathrm{DBO}_{5}$ dissoute. Le tableau 4 présente les teneurs et les proportions de la $\mathrm{DBO}_{5}$ particulaire dans les effluents issus des bassins facultatif et de maturation.

$\mathrm{La} \mathrm{DBO}_{5}$ particulaire atteint des valeurs beaucoup plus importantes dans le bassin facultatif que dans le bassin de maturation, respectivement $(10-235 \mathrm{mg} / \mathrm{L})$ et $(5-190 \mathrm{mg} / \mathrm{L})$.

Les concentrations résiduelles en $\mathrm{DBO}_{5}$ filtrée de l'effluent traité varient entre 10 et $220 \mathrm{mg} / \mathrm{L}$, à comparer au seuil maximum de $25 \mathrm{mg} / \mathrm{L}$ suggéré par la réglementation européenne (Council of the European Communities, 1991). En dépit des fortes charges algales souvent observées aussi bien dans le bassin facultatif que dans le bassin de maturation, la qualité de l'effluent à la sortie en matière de MES répond 
Tableau 4. Concentrations en $\mathrm{DBO}_{5}$ particulaire $\left(\mathrm{DBO}_{5} \mathrm{P}\right)$ et leur proportion par rapport à la $\mathrm{DBO} 5$ totale.

Table 4. Concentrations of particulate $\mathrm{BOD}_{5}$ (calculated as unfiltered $\mathrm{BOD}_{5}$ minus filtered.

\begin{tabular}{lcccc}
\hline & \multicolumn{2}{c}{$\mathbf{D B O}_{5} \mathbf{P} \mathbf{~ m g} / \mathbf{L}$} & \multicolumn{2}{c}{$\mathbf{D B O}_{5} \mathbf{P} / \mathbf{D B O}_{\mathbf{5}}$ Totale } \\
en $\%$ \\
Bassin & Intervalle & Moyenne & Intervalle & Moyenne \\
Bassin facultatif & $10-235$ & 66 & $9-84$ & 45 \\
Bassin maturation & $5-190$ & 55 & $4-85$ & 47 \\
\hline
\end{tabular}

fréquemment aux normes de rejet recommandées par la directive de l'Union Européenne $(<150 \mathrm{mg} / \mathrm{L})$. Ceci est le cas dans $76 \%$ des mesures effectuées $(n=75)$.

\subsection{2 Élimination des nutriments}

L'élimination de l'azote a été analysée sous trois formes, à savoir : l'azote total kjeldahl, l'azote ammoniacal et l'azote nitrique. Pour le phosphore, c'est le phosphore total et les ortho-phosphates qui ont été analysés. Les figures $6,7,8$ et 9 présentent respectivement les concentrations en phosphore et en azote (moyenne, minimum et maximum) mesurées durant la période de l'étude dans les effluents bruts (EB) et les effluents issus de chaque bassin (BA, BF et BM). Les figures 10 et 11 présentent respectivement l'évolution des rendements épuratoires en azote et en phosphore durant cette période.

Les teneurs résiduelles moyennes en phosphore total et ortho-phosphates de l'effluent à la sortie de la filière sont respectivement de $12,5 \mathrm{mg} / \mathrm{L}$ et $4,6 \mathrm{mg} / \mathrm{L}$. Ces valeurs sont très élevées par rapport à la limite tolérable de $0,1 \mathrm{mg} / \mathrm{L}$ en phosphore total pour un rejet d'effluents dans un milieu sensible à l'eutrophisation (AYERS et WESTCOT, 1985; WHO, 1989). Le niveau d'élimination du phosphore est faible et instable avec un rendement d'élimination moyen de 17,2\% pour le phosphore total et 19,2\% pour les ortho-phosphates. Des rendements négatifs en phosphore ont souvent été observés, notamment durant les périodes chaudes de mars à mai où il s'effectue des remontées de boues dans les bassins, et plus particulièrement dans le bassin anaérobie.

Les résultats des mesures des nutriments azotés présentent des niveaux d'abattement variés par bassin. C'est le cas par exemple de l'azote ammoniacal dont la teneur présente une légère augmentation dans le bassin anaérobie (Figure 8). Dans ce bassin, l'azote organique est hydrolysé en azote ammoniacal, la concentration en azote ammoniacal de l'effluent sortant peut être supérieure à celle de l'influent brut entrant. Dans notre étude, l'azote ammoniacal a évolué de $47,3 \mathrm{mg} / \mathrm{L}$ en moyenne dans l'influent brut à $51,2 \mathrm{mg} / \mathrm{L}$ en moyenne dans l'effluent à la sortie du bassin anaérobie, soit une augmentation moyenne d'environ 8,2 \% dans ce bassin. SILVA et al. (1995) et MARA et al. (2001) rapportent un pourcentage d'augmentation des teneurs en azote ammoniacal de 29,2 \% sur un bassin anaérobie au nord-est du Brésil. À l'inverse, dans le bassin de maturation, l'élimination de l'azote est de mise, avec un abattement de $29,8 \%$ en moyenne. En effet, les valeurs de $\mathrm{pH}$ variant de 7,8 à 10,3 dans ce bassin justifient amplement l'abattement de l'azote par «stripping». Pour un temps de rétention hydraulique (TRH) total de 18 jours, le rendement cumulatif en azote ammoniacal obtenu pour l'ensemble de la filière est évalué à 38,4\% en moyenne. Selon les données de littérature, le rendement d'élimination de l'azote ammoniacal dans une filière de lagunage à microphyte est très variable. Certains auteurs aboutissent à des valeurs de rendement plus faibles, moins de $10 \%$ (SILVA et al., 1987; TOMS et al., 1975) et d'autres à des valeurs élevées de l'ordre de $95 \%$ (MIDDLEBROOKS et al., 1982) en fonction de la configuration du système et des caractéristiques de fonctionnement des bassins en série. Les valeurs de rendement les plus élevées rapportées par MIDDLEBROOKS et al. (1982) sont expliquées par un TRH plus important (227 jours) dans une série de bassins de faible profondeur (1,2 m). SANTOS et OLIVEIRA (1987) ont obtenu au Portugal un rendement moyen annuel de 52,4\% sur une filière en série comprenant un bassin anaérobie (TRH de 1,7 jours), un bassin facultatif (TRH de 17,3 jours) et un bassin de maturation (TRH de 9,7 jours). SILVA (1982) rapporte sur une filière de cinq bassins en série (un bassin anaérobie, un bassin facultatif et trois bassins de maturation) des rendements de $32 \%$, $48 \%$, et $81 \%$ respectivement pour des TRH de 8,5 jours, 17 jours, et 29,1 jours.

La charge en nitrate del'influent brute entrant dans le système montre des concentrations variant de $1,10 \mathrm{mg} / \mathrm{L}$ à $13,10 \mathrm{mg} / \mathrm{L}$ avec une moyenne de $4,03 \mathrm{mg} / \mathrm{L}$. Selon METCALF et EDDY (1991), les nitrates sont typiquement absents dans les effluents domestiques non traités. Dans notre cas, leur présence en faible concentration pourrait être le fait d'une légère nitrification de l'effluent dans le réseau de conduite en écoulement gravitaire, et éventuellement dans la bâche de relevage d'où sont collectées les eaux usées non traitées avant être pompées vers le bassin anaérobie. Le long de la filière, l'évolution des teneurs en nitrate montre une diminution allant de $4 \mathrm{mg} / \mathrm{L}$ en moyenne dans l'effuent brut à 2,2 mg/L dans l'effluent traité. Cette évolution en décroissance des teneurs en nitrate semble être en accord avec les résultats de FERRARA et ACVI (1982) qui ont démontré avec évidences que, normalement, la nitrification ne se réalise pas de manière sensible dans les bassins de stabilisation.

Quant à l'efficacité globale du système en matière d'élimination de l'azote total kjeldahl (NTK), les mesures ont abouti à des performances moyennes d'élimination de $76 \%$. Ce résultat est de même ordre de grandeur que ceux suggérés par différents auteurs (70 \% à $90 \%$ ) (MARA et PEARSON, 1998). L'effluent traité présente en sortie des teneurs moyennes 


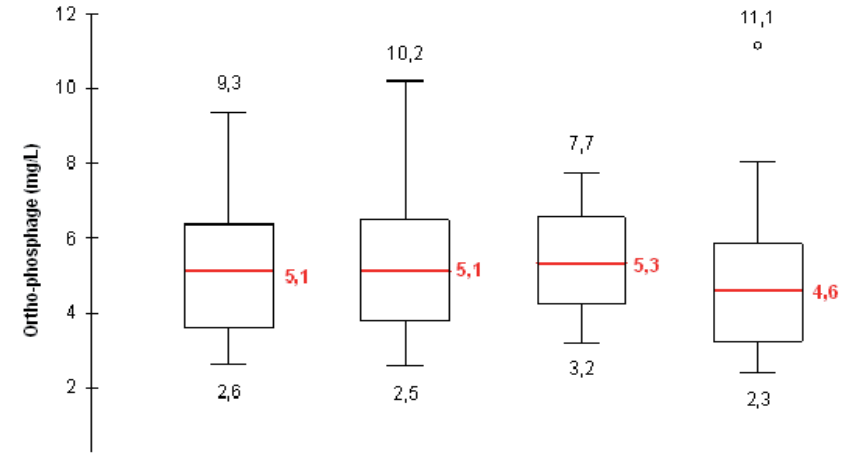

Figure 6. Variation des teneurs en ortho-phosphates à travers le système.

Ortho-phosphates variation throughout the system.
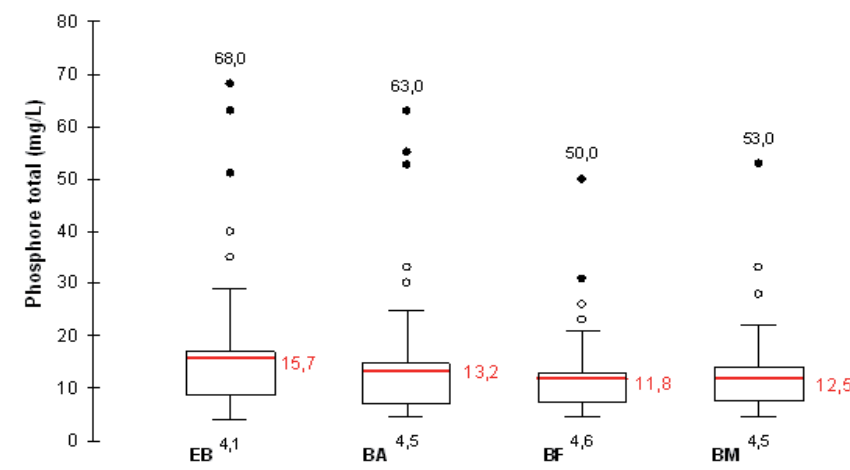

Figure 7. Variation des teneurs en phosphore total à travers le système.

Total phosphorus variation throughout the system.
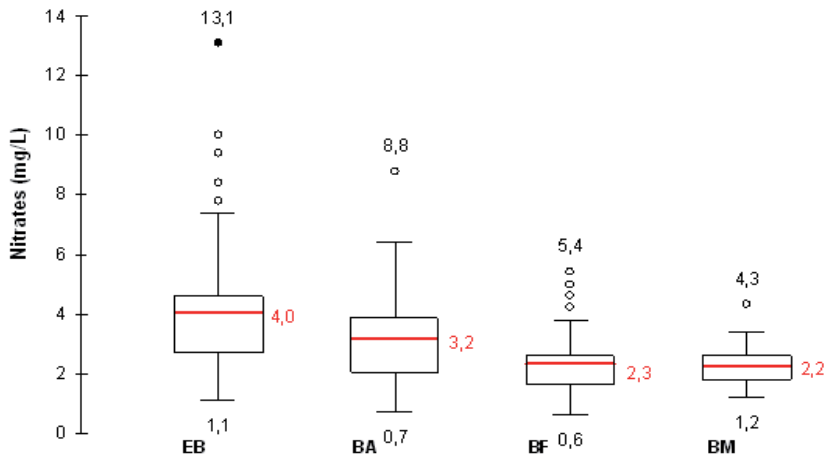

Figure 9. Variation des teneurs en nitrate à travers le système. Nitrate nitrogen variation throughout the system.

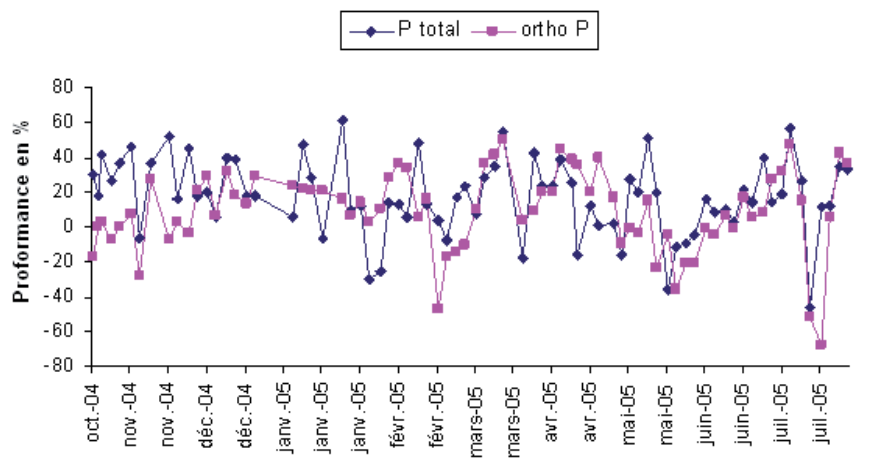

Figure 10. Rendements épuratoires en phosphore durant la période de l'étude.

Phosphorus removal efficiency during the study period.
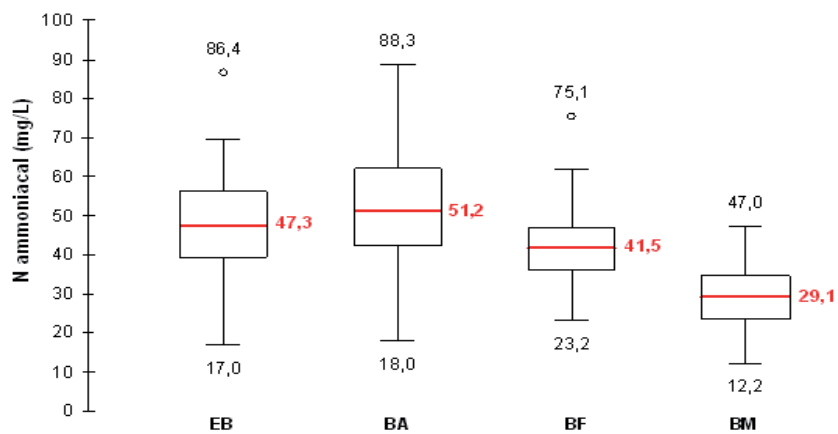

Figure 8. Variation des teneurs en azote ammoniacal à travers le système.

Ammonia nitrogen variation throughout the system.

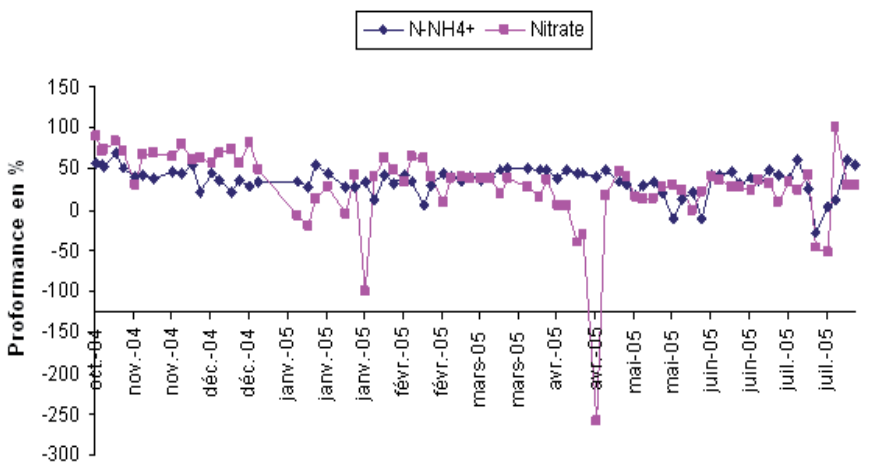

Figure 11. Rendements épuratoires en azote ammoniacal et en nitrate durant la période de l'étude. Ammonia and nitrate nitrogen removal efficiencies during the study period. 
en azote total kjeldahl de l'ordre de 47,37 mg/L. Cette teneur résiduelle est très élevée par rapport à la limite tolérable de $5 \mathrm{mg} /$ L d'azote total kjeldahl proposée par AYERS et WESTCOT (1985), et l'OMS (1989) pour un rejet d'effluent dans les écosystèmes sensibles à l'eutrophisation.

3.2.3 Élimination de la pollution bactérienne et parasitaire

L'abattement de la pollution bactérienne et parasitaire a été étudié avec comme indicateurs d'une part, les coliformes fécaux, et d'autre part, les oufs d'helminthes et les kystes de protozoaire. Le tableau 5 présente les valeurs moyennes, maxima, minima d'Unités Format Colonies (UFC). Les mesures ont été effectuées sur 47 échantillons durant la période de l'étude sur l'eau brute et l'effluent à la sortie de chaque bassin.

Les eaux usées brutes présentent des charges en coliformes fécaux variant de $2 \times 10^{5}$ à $8,6 \times 10^{7}$ ufc avec une valeur moyenne de 1,6 $\times 10^{7}$ ufc. Cette charge est du même ordre de grandeur que celle généralement rencontrée dans les effluents urbains (LAZAROVA et al., 1998; NIGIM et al.; 2002). Sur la base de la classification adoptée par METCALF et EDDY (1991), cette charge correspondrait à la composition bactériologique type des effluents bruts d'eaux usées de la catégorie de charge faible à moyenne. Dans l'effluent traité à la sortie de la filière, la teneur résiduelle en coliformes fécaux est en moyenne de $5,4 \times 10^{3} \mathrm{ufc}$, avec un minimum de $1 \times 10^{3} \mathrm{ufc}$ et un maximum de $3,4 \times 10^{5}$ ufc. Il a ainsi été enregistré au cours de la période de l'étude des valeurs élevées d'abattement de la charge en coliformes fécaux avec un maximum de 4,9 ulog en mai, période de forts ensoleillements et de grandes températures. Selon les directives de l'OMS, les eaux épurées ne peuvent faire l'objet de réutilisation en irrigation non restrictive que pour des charges en coliformes fécaux inférieures à $1000 \mathrm{ufc} / 100 \mathrm{~mL}$ (WHO, 1989). Ainsi, les résultats issus de cette étude menée sous climat sahélien restent peu satisfaisants pour l'élimination des coliformes fécaux (selon cette directive) malgré un temps de séjour moyen de 18 jours dans les trois bassins. Les résultats de cette étude sont toutefois légèrement meilleurs par rapport à ceux obtenus par MADERA et al. (2002) sur une filière similaire en climat tropical humide en Colombie. Avec un temps de séjour total de 12 jours, les auteurs rapportent des concentrations résiduelles en coliformes fécaux de l'ordre 6,6 × $10^{3}$ ufc.

Sur le plan de la pollution parasitaire, les analyses ont révélé sur les eaux usées brutes, la présence d'œufs d'helminthes parasites (Ankylostome, Ascaris, Tricocéphale), des larves d'anguillules et des kystes de protozoaires (Entamoeba coli, Entamoeba histolytica). La charge de l'eau brute en œufs d'helminthes et de larves est faible, de l'ordre de 1 en moyenne par litre. Pour les kystes d'ascaris, la valeur obtenue est de deux unités en moyenne par litre, tandis que pour les kystes d'Entamoeba coli, les valeurs obtenues varient de 6 à 272 kystes par litre. Le tableau 6 présente les valeurs moyennes pour les indicateurs de pollution parasitaire.

On peut noter qu'au stade primaire du traitement déjà (au niveau du bassin anaérobie) les œufs d'Ankylostomes et de

Tableau 5. Variation des coliformes fécaux ( UFC/100 mL ) à travers le système.

Table 5. Results for faecal coliforms (CFU/100 $\mathrm{mL}$ ) in samples of raw sewage water and effluent from each pond.

\begin{tabular}{lccc}
\hline \multicolumn{1}{c}{ Échantillon } & $\begin{array}{c}\text { Moyenne } \\
\text { géométrique }\end{array}$ & Intervalle & n \\
\hline Eaux usées brutes & $1,6 \times 10^{7}$ & $2 \times 10^{5}-8,6 \times 10^{7}$ & 47 \\
Effluent Bassin Anaérobie & $2,6 \times 10^{6}$ & $1,2 \times 10^{5}-7,5 \times 10^{6}$ & 47 \\
Effluent Bassin Facultatif & $4,1 \times 10^{5}$ & $4 \times 10^{4}-1,7 \times 10^{6}$ & 47 \\
Effluent Bassin Maturation & $5,4 \times 10^{3}$ & $1 \times 10^{3}-3,4 \times 10^{5}$ & 47 \\
\hline $\mathrm{n}=$ nombre d'échantillons analysés & & &
\end{tabular}

Tableau 6. Évolution des concentrations en parasites à travers le système.

Table 6. Concentration of parasites (belminth eggs and protozoa cysts) in raw wasterwater and effluent from each pond.

\begin{tabular}{|c|c|c|c|c|c|c|c|c|c|}
\hline \multicolumn{2}{|c|}{ Parasites } & \multicolumn{2}{|c|}{ Eaux brutes } & \multicolumn{2}{|c|}{ Bassin Anaérobie } & \multicolumn{2}{|c|}{ Bassin Facultatif } & \multirow{2}{*}{$\begin{array}{c}B M \\
\text { moyenne }\end{array}$} & \multirow[b]{2}{*}{$n$} \\
\hline & & Moyenne & Intervalle & Moyenne & Intervalle & Moyenne & Intervalle & & \\
\hline Ankylostome & (œuf/litre) & 1 & $0-4$ & 0 & 0 & 0 & 0 & 0 & 25 \\
\hline Ascaris & (œuf/litre) & 2 & $0-10$ & 1 & $0-4$ & 1 & $0-1$ & 0 & 25 \\
\hline Tricocéphale & (œuf/litre) & 1 & $0-1$ & 0 & 0 & 0 & 0 & 0 & 25 \\
\hline Anguillule & (larve/litre) & 1 & $0-5$ & 0 & 0 & 0 & 0 & 0 & 25 \\
\hline E histolytica & (kyste/litre) & 21 & $2-55$ & 1 & $0-3$ & 0 & 0 & 0 & 25 \\
\hline
\end{tabular}

$\overline{\mathrm{BM}}=$ Bassin de maturation, $\mathrm{E}$ coli= entamoeba coli, E histolitica= Entamoeba histolitica. 
Tricocéphale sont entièrement éliminés, les autres parasites y sont partiellement éliminés à hauteur de $60 \%$ à $100 \%$ pour les œufs d'ascaris, $80 \%$ à $100 \%$ pour les kystes d'Entamoéba coli et $90 \%$ à $100 \%$ pour les kystes d'Entamoeba Histolitica. Sur l'ensemble des échantillons analysés $(\mathrm{n}=25)$, l'effluent à la sortie de la filière est resté exempt de parasites, soit un rendement constant de $100 \%$. La nouvelle filière de trois bassins en série (avec un traitement primaire dans un bassin anaérobie) a permis d'obtenir des rendements optimaux d'élimination de la charge parasitaire par rapport à une ancienne configuration de deux bassins en séries testée par KLUTSÉ et al. (1995) dans les mêmes conditions climatiques sahéliennes. Sur des eaux usées domestiques préalablement traitées par un décanteur primaire puis soumises à épuration pendant 16,2 jours dans une série de lagunage (constituée d'un bassin facultatif et d'un bassin de maturation) l'auteur rapporte des rendements globaux de $94 \%$ d'élimination des œufs d'helminthe et de kyste de protozoaire. Lapproche évolutive dans la conception des installations dans notre étude montre bien l'importance d'un traitement primaire dans un bassin anaérobie dans la réduction de la pollution parasitaire sous climat sahélien. Vu les teneurs résiduelles en coliformes fécaux, l'effluent en sortie de la filière de trois bassins en série de 2IE peut être classé en catégorie B (recommandation de l'OMS pour la réutilisation agricole des eaux usées, 1989); C'est-à-dire que cette réutilisation ne peut être envisagée que pour les céréales, les fourrages, les arbres fruitiers et les cultures industrielles.

\section{CONCLUSION}

L'étude a permis d'évaluer les performances épuratoires d'une série de trois bassins de lagunage à microphytes sous climat sahélien. Les résultats présentent des rendements épuratoires satisfaisants pour l'élimination des matières en suspension en accord avec les normes de rejet des effluents de lagunage recommandées par l'Union Européenne. L'élimination de la $\mathrm{DBO}_{5}$ reste partielle et les valeurs résiduelles de la $\mathrm{DBO}_{5}$ filtrée sont en grande partie au-dessus des niveaux recommandés par l'Union Européenne.

L'élimination des nutriments (en azote et en phosphore) est très faible. Les concentrations résiduelles restent très élevées dans l'effluent traité et pourraient constituer un grand risque d'eutrophisation pour des rejets dans un écosystème aquatique. Cependant, ils constitueront un apport intéressant de fertilisation en nutriments azotés et phosphorés dans le cas d'une réutilisation en agriculture urbaine.
L'abattement de la pollution bactérienne est peu satisfaisant. La charge résiduelle moyenne en coliformes fécaux dans l'effluent traité reste encore relativement élevée $\left(5,4 \times 10^{3} \mathrm{ufcl}\right.$ $100 \mathrm{ml})$ par rapport à la directive OMS ( $\leq 1000 \mathrm{ufc} / 100 \mathrm{~mL})$ pour une irrigation non restrictive. Le rendement épuratoire enregistré est de $100 \%$ pour l'élimination des parasites (œufs d'helminthes et kystes de protozoaire). L'absence totale de parasites dans l'effluent traité durant toute la période de l'étude permet de le classer en catégorie B du classement OMS des eaux, pour une irrigation restrictive notamment. Elles sont donc seulement adaptées pour des produits destinés au bétail ou pour des cultures fruitières. Pour des usages qui sortent de cette catégorie comme le maraîchage (activité couramment menée à Ouagadougou), il convient de mettre en place un système de traitement quaternaire comme la filtration sur gravier après le bassin de maturation. Le 2IE entame cette étude dès l'année 2007.

\section{RÉFÉRENCES BIBLIOGRAPHIQUES}

ABIS K.L. (2002). The performance of facultative waste stabilisation ponds in the United Kingdom. Thèse de doctorat, School of Civil Engineering, University of Leeds, 212 p.

ARTHUR J.P. (1983). Notes on the design and operation of waste stabilization ponds in warm climates of developing countries. Rapport technique № 7, Washington, DC, The World Bank.

AYERS R.S. et D.W. WESTCOT (1985). Water quality for agriculture. FAO Irrigation and Drainage Paper No 29. United Nations Food and Agriculture Organization, Rome, Italie.

COUNCIL OF THE EUROPEAN COMMUNITIES (1991). Directive du conseil 91/271/EEC du 21 mai 1991 concernant le traitement des eaux usées urbaines. Off. J. Eur. Communities, L135, 40-52 (30 mai).

http://www.europa.eu.int/comm/environment/water/ water-urbanwaste/directiv.html

CIEH (1993). Comité inter-africain d'études hydrauliques «Étude comparative des systèmes d'épuration collectifs dans le contexte africain». CIEH, Ouagadougou, Burkina Faso, 66 p.

FERRARA R.A et C.B. AVCI (1982). Nitrogen dynamics in waste stabilization ponds. J. Water Pollut. Contr. Fed., 54,4, 361-369. 
GLS, société d'ingénierie et de conseils. La biodégradabilité des effluents urbains, memotec 19 http://www.gls.fr/ memotec19.htm (consultation le 12juillet 2006).

GUÈNE O. (1989). Contribution à l'étude du fonctionnement et de la modélisation de l'épuration de la pollution carbonée et microbienne des lagunes naturelles sous climat sahélien. Cas de la station pilote de l'EIER. Rapport de recherche / EIER. $65 \mathrm{p}$.

KLUTSÉ A. (1995). Épuration des eaux usées domestiques par lagunage en zone soudanosahélienne (Ouagadougou, Burkina Faso). Thèse de doctorat, Université Montpellier II, 160 p.

\section{LAZAROVAV., P.SAVOYE, M.L.JANEX,E.R. BLATCHLEY} et M. POMMEPAY (1998). Advanced wastewater disinfection technologies: State of the art and perspectives. $2^{\text {e }}$ International conference on advanced wastewater treatment, recycling and reuse, $\mathrm{N}^{\circ} 6,14-16$ septembre 1998, Milan, Italie, 354-362.

MADERA C.A., M.R. PENA, et D.D. MARA (2002). Microbiological quality of a waste stabilisation pond effluent used for restricted irrigation in Valle Del Cauca, Colombia. Water Sci. Tech., 45,1, 139-143.

MARA D.D et H.W. Perason (1998). A design manual for waste stabilization ponds in Mediterranean Countries. Lagoon technology international, Leeds, UK, xiv + 112 p.

MARA D.D. (1976) Sewage treatment in hot climate. John Wiley and Sons, Chichester, UK.

MARA D.D et H.W. PEARSON (1986). Artificial freshwater environments: Waste stabilization ponds. Dans: Biotechnology, vol. 8, SCHOENBORN W. (Éditeur)), pp. 177-206. Weinheim: VVCH Verlagsgesellschaft.

METCALFANDEDDY, INC. (1991). Wastewaterengineering: Treatment, disposal and reuse, $3^{\mathrm{e}}$ éd., MCGRAW-HILL INTERNATIONAL, N.Y., États-Unis.

MIDDLEBROOKS E.J., C.H. MIDDLEBROOKS, J.H. REYNOLDS, G.Z. WATERS, S.C. REED et D.B. GEORGE (1982). Wastewater stabilization lagoon design. Performance and upgrading. Macmillan, N.Y., États-Unis.

NIGIM H.H., W.A. HASHLAMOUN, J.Y. AL-DADAH et J.L. VASEL (2002). Potential implication of subsurface drip irrigation with primary treated wastewater. IWA regional symposium on water recycling in Mediterranean region. Iraklio, Grèce, 26-29 septembre 2002. Préimprimé, 2, 171-174.
RADIN N (1990). Analyse critique du fonctionnement de la station d'épuration de l'EIER, performances de la filière des lits bactériens. Travail pratique de diplôme, EPFL, Suisse, $71 \mathrm{p}$.

SILVA S.A. (1982). On the treatment of domestic sewage in waste stabilization ponds in northeast Brazil. Thèse de doctorat, University de Dundee, UK.

SILVA S.A., D.D. MARA et R. DE OLIVEIRA (1987). The performance of five deep waste stabilization ponds in northeast Brazil. Water Sci. Tech., 19,12, 123-130.

TOMS I.P., M. OWENS, J.A. HALL et M.J. MINDENHALL (1975). Observation on the performance of polishing lagoons. Water Pollut. Contr., 74, 383-401.

WHO (1989). Health guidelines for the use of wastewater in agriculture and aquaculture. Rapport technique, série $\mathrm{N}^{\circ}$ 778, World Health Organization, Genève, Suisse. 